\title{
Shaping the waves
}

An optical compensation technique enables deeper, crisper two-photon microscopy.

A typical commercial two-photon microscope achieves imaging depths of about 500 microns in mammalian tissue, but at this depth, fine details are hard to see. Upon his arrival at the Janelia Farm Research Campus, in Virginia, Meng Cui's goal was to develop technology that would allow scientists to obtain better images at larger depths.

Cui set out to use optical tricks to compensate for the aberrations and scattering distortions that deteriorate an image by decreasing the number of light rays that reach a distant focus position. To do this, he and his colleagues iteratively changed the wavefront of the illuminating light while continuously monitoring the light power of the output signal-here the two-photon fluorescence of the imaging target-until they found the configuration that produced the best focus.

The team used a segmented deformable mirror, based on micromechanical systems, that provides many individually controlled segments for the modulation of the incoming light. They divided the segments into two halves: one half was kept stationary-and served as the reference-and the other half was modulated simultaneously. This process was iteratively repeated until the signal at the focus was maximized.

The researchers named the method IMPACT (iterative multiphoton adaptive compensation technique), and they used it to image layer- 5 neurons expressing GFP in a 400-micron-thick slice of fixed mouse brain tissue as well as GFP-labeled T cells residing 800 microns deep inside fixed lymph nodes. The group also imaged fluorescence beads inserted into lymph nodes in a living mouse.

In contrast to adaptive optics-based methods for correcting optic distortions, IMPACT does not need an image to start with: "If you are working at this depth, you don't really have an image in the first place," Cui says. IMPACT also has advantages in terms of speed and signal power, and because the light is directed more efficiently to the focus, the amount of laser power required for excitation is reduced, thus diminishing phototoxicity and photodamage to the sample.

IMPACT's major limitation right now is that each wavefront correction enables imaging of only a small field of view (an area of about 10 microns), and multiple images have to be stitched together to see the larger picture. With Cui already working to overcome this limitation, this method has the potential to be true to its name and change the way we see through living tissues.

\section{Erika Pastrana}

\section{RESEARCH PAPERS}

Tang, J. et al. Superpenetration optical microscopy by iterative multiphoton adaptive compensation technique. Proc. Natl. Acad. Sci. USA 109, 84348439 (2012). 